\title{
Imaginario colectivo, movimientos juveniles y cultura ciudadana juvenil en El Salvador: análisis de percepción de derechos
}

\section{Social imaginary, youth movements and youth citizen culture in El Salvador: An analysis on the perception of rights}

\author{
Saúl Campos-Morán' \\ saul.campos@utec.edu.sv \\ Paola María Navarrete ${ }^{2}$ \\ Carlos Felipe Osegueda ${ }^{3}$ \\ Investigadores de la Utec
}

Recibido: 29/04/2015 - Aceptado: 06/06/2015

\section{Resumen}

El objetivo de la investigación fue identificar los elementos culturales, de cosmovisión, de conceptos en el imaginario colectivo, actitudes y contextos educativos que inciden en la formación y participación ciudadana de los jóvenes salvadoreños. La investigación fue de tipo ex post facto, con diseño transversal (Montero y León, 2007), constituida del diagnóstico de una situación ya existente a partir de una hipótesis dada, sin tener la oportunidad de manipular la variable independiente utilizando un post-test sin grupo control. Esto se complementó con técnicas cualitativas de recolección de información, utilizando la entrevista estructurada como técnica de apoyo. Entre los principales resultados se tiene que en el país existen más ventajas de formación para los hombres que para las mujeres; que existen diferencias

\section{Abstract}

The aim of this research was to identify the cultural elements, the worldview and the concepts in the social imaginary; these attitudes and contexts influence the shaping and participation of young Salvadorans as citizens. The research was ex post facto type with cross design (Montero and León, 2007); it is based on the diagnosis of a pre-existing situation and a given hypothesis; there was no opportunity to manipulate the independent variable using a post-test without a control group. This was complemented with qualitative data collection techniques, using the structured interview as technical support. Among the main results there can be mentioned that there are more training advantages for men than for women; there are differences in the type of participation exercised based on the area of residence;

Antropólogo de profesión, Máster en Docencia e Investigación Educativa y doctorando en Proyectos, investigador y docente en la Universidad Tecnológica de El Salvador. Correo electrónico saul.campos@utec.edu.sv

Antropóloga, investigadora de la Universidad Tecnológica de El Salvador y docente en la Escuela de Antropología. Correo electrónico paola.navarrete@utec.edu.sv Antropólogo, docente investigador de la Escuela de Antropología de la Universidad Tecnológica de El Salvador. Correo electrónico carlos.osegueda@utec.edu.sv 
entre el tipo de participación que se puede ejercer según el área de residencia, y que el tipo de educación recibida es determinante para las competencias de participación ciudadana que los jóvenes ejercen.

\section{Palabras clave}

Participación ciudadana, juventud, política, movimientos juveniles also, the type of education received is crucial in terms of the citizen participation competencies that youths exercise.

\section{Keywords}

Citizen participation, youth, politics, youth movements.

\section{Introducción}

Campos (2011) encontró que en El Salvador existe juventud ávida de participar en el rumbo de su país; pero que no cuenta con las herramientas conceptuales para hacerlo a través de los espacios de participación tradicionales ni con las posibilidades de participación que se tienen respecto al nivel económico y educativo de los jóvenes. De tal forma, es imperativo definir las implicaciones de este fenómeno social a través de algunos elementos centrales, que, según los resultados de la investigación sobre juventud y democracia realizada por la Utec en el 2011, se identificaron como factores: democracia, ciudadanía, cultura y educación. Las relaciones, que para efectos del estudio se supone existen entre estos elementos, son las siguientes: tal como ya fue señalado, siendo un país cuyo $48 \%$ de la población es menor de 25 años, la consolidación democrática salvadoreña requiere de ciudadanos jóvenes dispuestos a cumplir con sus papeles de forma activa en los espacios ciudadanos; la ciudadanía es un fenómeno que se construye a partir de la sociedad misma; las personas de una sociedad requieren ser formadas como ciudadanos, lo que implica revisar los papeles que desempeñan la escuela y el hogar, pero principalmente el papel de la sociedad misma en la formación de sus ciudadanos; la educación de los jóvenes para formar futuros ciudadanos se realiza a través de contenidos impartidos por medio de agentes, tanto formales como no formales, y es impartida desde diferentes agentes socializadores que son sus responsables, entre estos están la escuela y la universidad; la cultura colectiva y el imaginario colectivo juvenil del lugar de los jóvenes en la estructura política y ciudadana de nuestro país.
A partir de lo anterior, el problema de estudio se define de la siguiente manera: el concepto de democracia, así como los de ciudadanía y participación, son fenómenos múltiples, dinámicos, inter-subjetivos y relativos al contexto histórico. Por tanto, es posible que estos sean interpretados de diferentes formas (Vuanello, 2007). Se parte del supuesto de que la participación política y ciudadana es un elemento que está culturalmente configurado como apático, lo que se refleja en el poco entusiasmo de participar; en las actitudes negativas al involucramiento en temas de país y a la consideración del mundo oficial, como una realidad aparte en el día a día de los jóvenes (Campos, 2011). Se puede apreciar que dentro del imaginario colectivo de la juventud salvadoreña se encuentra de manera explícita la idea de ciudadanía, que emerge desde distintos entornos y sectores sociales con diferentes significados. Si consideramos que estos llevan a tendencias de acción (Campos, 2011), entonces podemos presumir que la falta de contenidos claros y explícitos repercutirá negativamente en la existencia de estrategias consistentes para la formación ciudadana $y$, consecuentemente, en el éxito del proceso de consolidación democrática. Además, pareciera que una democracia fuerte y estable necesita de un alto grado de coherencia entre los ideales nacionales y los educacionales (Fernández, 2001).

Toda esta situación obliga a buscar identificar y conocer los diferentes elementos constituyentes de la cultura ciudadana, que, desde diferentes instancias, tienen poder de influencia en la juventud, y son asociados por esta al concepto de ciudadanía, distinguiendo aquellos frente a los cuales existen consensos, desacuerdos y contradicciones. Seguidamente, también es necesario revisar el papel de 
la juventud y la responsabilidad que la sociedad tiene y otorga a los agentes socializadores en la formación cívica de estos.

Datos del Tribunal Supremo Electoral (2014) demostraron que entre la población salvadoreña existe un gran nivel de desinterés por los asuntos políticos y poca voluntad de ejercer su ciudadanía democrática, comprobando por la baja participación electoral que se tuvo durante las elecciones presidenciales de 2014. Este dato evidencia el problema de estudio, el cual aparece especialmente relevante considerando los contextos nacionales e internacionales que se están viviendo.

A escala nacional, la transición hacia la democracia se ha visto enfrentada a la reaparición de temas ligados a los derechos humanos, la memoria histórica y las responsabilidades que le caben a distintos actores, particularmente al Estado, las Fuerzas Armadas, los partidos políticos y la sociedad civil, en la definición de este nuevo escenario. Los interrogantes sobre el papel que debiera jugar cada uno de estos actores se han visto reforzados con el ambiente de preparación y la coyuntura política de las elecciones presidenciales recién pasadas.

A escala internacional, la llamada "globalización", junto con una era de revolución de las tecnologías y las comunicaciones, se ha traducido en inevitables cambios en las formas de relacionarse y de convivir en espacios públicos. Además, han resituado el papel y el modo de acceder a la información y al conocimiento; y han cuestionado los límites o relaciones existentes entre lo público y lo privado, así como también entre lo nacional/local y lo internacional/ global.

Por otra parte, y aludiendo al tema de la participación juvenil a escala mundial, las contradicciones entre el desarrollo sostenido y la equidad han puesto a la formación ciudadana frente al desafío de aportar a la conciliación de ambos aspectos, encontrando una nueva relación entre desarrollo y democracia.
En definitiva, tanto el escenario nacional como el internacional dan cuenta de la necesidad de revisar la forma en que tradicionalmente han sido concebidos conceptos como ciudadanía y el papel de la educación en la consolidación de su ejercicio.

De tal manera, se planteó como objetivo de la investigación el identificar los elementos culturales, de cosmovisión, de conceptos en imaginario colectivo, actitudes y contextos educativos que inciden en la formación y participación ciudadana de los jóvenes salvadoreños, y crear una red temática de democracia en base a su identificación. Asimismo, entre los objetivos específicos se planteó conocer el significado de ciudadanía y democracia en el imaginario colectivo de los jóvenes salvadoreños, así como determinar la incidencia de la educación formal en la noción de ciudadanía de los jóvenes salvadoreños; identificar elementos de la vida cotidiana y la cosmovisión juvenil que inciden en la cultura ciudadana de los sujetos; conocer la incidencia de los grupos sociales de pertenencia de los jóvenes en su participación ciudadana y determinar si existen diferencias entre sectores sociodemográficos en cuanto a la formación y la participación ciudadana.

\section{Método}

\section{Participantes}

Para participar en el estudio se tomó en cuenta a jóvenes salvadoreños entre 15 y 24 años, masculinos y femeninos, de todos los niveles educativos con residencia tanto urbana como rural, sin importar preferencia política, de los catorce departamentos de El Salvador.

\section{Universo y muestra}

Según datos de la Encuesta de Hogares y Propósitos Multiples (EHPM) 2013, el total de jóvenes de entre 15 y 24 años de El Salvador suman el 20 \% de la población nacional. A partir de estos datos se hizo un cálculo del $95 \%$ de nivel de confianza y $3 \%$ de margen de error, resultando en una muestra de 1.020 sujetos. 
Tabla 1.

Distribución de la muestra según porcentajes por departamento

\begin{tabular}{clcc}
\hline No. & \multicolumn{1}{c}{ Departamento } & $\%$ & Muestra $(\mathbf{n}=1.020)$ \\
\hline 1 & Ahuachapán & 5 & 65 \\
2 & Santa Ana & 8 & 103 \\
3 & Sonsonate & 7 & 90 \\
4 & Chalatenango & 3 & 40 \\
5 & La Libertad & 11 & 142 \\
6 & San Salvador & 32 & 421 \\
7 & Cuscatlán & 4 & 52 \\
8 & La Paz & 5 & 65 \\
9 & Cabañas & 2 & 26 \\
10 & San Vicente & 3 & 40 \\
11 & Usulután & 6 & 77 \\
12 & San Miguel & 7 & 90 \\
13 & Morazán & 3 & 40 \\
14 & La Union & 3 & 40 \\
\hline
\end{tabular}

Fuente: Elaboración propia en base a datos de la EHPM (2013) y datos calculados para el estudio $(Z=1.96)$

\section{Descripción de la población encuestada}

En cuanto a las frecuencias de los datos sociodemográficos de la población sondeada (ver tabla 2), se tiene que 504 encuestados $(49,4 \%$ ) son del sexo masculino, mientras que $516(50,6 \%)$ corresponden al sexo femenino. Asimismo, respecto a la edad de los participantes, se tiene que el 1,5\% tiene los 15 años de edad $(n=20)$, mientras que $8,9 \%$ tiene $16(n=115) ; 38 \%$ manifestó tener $17(n=391) ; 35,5 \%$ tenía $18(n=358) ; 9,2 \% 19(n=119) ; 4,8 \%$ tiene $20(n=62) ;$ y $2,0 \%$ participó en la investigación, con 21 años $(n=26)$. En base a estos datos, la media general de edad de la muestra es de 19.6 años.

Además del sexo y la edad, se recabaron las variables: completó su último nivel de estudios, área de vivienda, sector en que realizó sus estudios y trabaja, con el fin de hacer las pruebas de hipótesis respectivas. En cuanto a las frecuencias para completó su último nivel de estudios, se tiene que el $87,4 \%$ de la población completó el último grado académico que cursó, mientras que el 12,6 \% abandonó sus estudios en algún momento; el $54,5 \%$ de la población reside en el área urbana, mientras que el $45,5 \%$ en la rural. En cuanto al sector en dónde realizó sus estudios de educación básica y media, se observa que el 78,2 \% estudió en el sector público, mientras que el 21, 8 \% manifestó haber estudiado en el sector privado de educación.

En cuanto a la cuestión de que si disponía de un trabajo al momento de participar en el estudio, se observa que el $26,1 \%$ de los participantes manifestó sí disponer de un trabajo, mientras que el 73,9 \% dijo no estar trabajando. Entre los que contestaron haber laborado, y en relación con los años que llevaba trabajando al momento de participar en el estudio, se observa que el $47,6 \%$ del total de los encuestados Ilevaba trabajando un año o menos al momento de participar; un 32,9 \% tenía entre uno y dos años; 3,5 \% tenía entre dos y tres años; y un 11,3\% tenían entre 3 y 4 años. Los que tenían entre 4 y 5 años fueron el $2 \%$; los de 5 a 6 años resultaron ser el 0,9\%; y los que tenían más de 6 años, es decir, habrían iniciado a trabajar desde una muy temprana edad, suman el $1,8 \%$.

Así mismo, y en concordancia con uno de los objetivos de la investigación, se sondeó la preferencia política de la población. A este respecto, un 59,2 \% de la población total 
manifestó no identificarse con ninguna corriente política. Los que dijeron ser de izquierda, por su parte, suman un $15,7 \%$, siendo los de derecha los que ocupan la segunda posición, con un $12,5 \%$. Los que dijeron ser de centro apenas suman $0,8 \%$ del total.

Tabla 2.

Preferencia político-ideológica de los participantes.

\begin{tabular}{|c|c|c|c|c|c|}
\hline & & Frecuencia & Porcentaje & $\begin{array}{l}\text { Porcentaje } \\
\text { válido }\end{array}$ & $\begin{array}{l}\text { Porcentaje } \\
\text { acumulado }\end{array}$ \\
\hline & Izquierda & 160 & 15,7 & 17,8 & 17,8 \\
\hline & Centro & 8 & 0,8 & 0,9 & 18,7 \\
\hline \multirow[t]{3}{*}{ Válidos } & Derecha & 128 & 12,5 & 14,2 & 32,9 \\
\hline & Ninguna & 604 & 59,2 & 67,1 & 100,0 \\
\hline & Total & 900 & 88,2 & 100,0 & \\
\hline Perdidos & Sistema & 120 & 11,8 & & \\
\hline Total & & 1.020 & 100,0 & & \\
\hline
\end{tabular}

También se les preguntó con quién vivían al momento de participar en el estudio. A este respecto, se observa que 49,8 \% manifestó residir con sus dos padres; seguido de $29,7 \%$ que dijo vivir en la casa de su madre; 7,8 \% que dice que vivía con los abuelos; $3,5 \%$ con el padre; $2,8 \%$ con los tíos; y $0,4 \%$ que dice vivir solo. Esto llama especialmente la atención, siendo que, a pesar de que parte de la muestra la conforman individuos mayores de 18 años, algunos llegando a los 24 años, la cantidad relativa de participantes que viven por su cuenta es muy pequeña, posiblemente evidenciando la conformación actual de los lazos matrilocales (término antropológico que denota que en un espacio determinado el mandato reside en la mujer) de la familia salvadoreña.

\section{Diseño y tipo de estudio}

La investigación fue de tipo ex post facto con diseño transversal (Montero y León, 2007), constituida del diagnóstico de una situación ya existente a partir de una hipótesis dada, sin tener la oportunidad de manipular la variable independiente utilizando un post-test sin grupo control. Esto se complementó con técnicas cualitativas de recolección de información, utilizando la entrevista estructurada como técnica de apoyo.

\section{Instrumento de recolección de datos}

El instrumento utilizado fue un cuestionario tipo encuesta, de elaboración propia, construido en escala Likert, con cuatro niveles que incluyeron desde $1=$ nada, $2=$ poco, 3 = bastante, 4 = completamente; y de escala dicotómica de sí y no. El instrumento sondea ítems standalone de naturaleza descriptiva, así como escalas de factor para los análisis inferenciales, representadas por las subescalas del instrumento; y una sección de pregunta abierta que sondeó la opinión general acerca del tema indagado. Así mismo, se realizó una guía de entrevista que sondeó ítems vinculados con la participación en la formación de ciudadanos de las ONG participantes del estudio.

Para determinar las escalas de factor se partió de la identificación de las variables que, según los objetivos, se planteó sondear en el instrumento. A partir de la realización de una prueba piloto donde participaron 160 individuos, se logró hacer una validación sistémica de la prueba, identificando cinco grandes factores sociales vinculados con la comprensión del tema. Entre los primeros están percepción del país, percepción de la condición de juventud y perspectivas del país, que se ordenaron en tres grupos; y las competencias de conocimiento, actitud, participación y formas de participación, que se desarrolló cada una en su propio factor.

\section{Resultados}

\section{Percepción de derechos y cultura ciudadana}

En primer lugar, se aprecia que para el ítem me interesa la situación política del país hay una media masculina de 3.31, 
un valor mayor al femenino, que alcanza el 3.03, siendo ambos ubicados dentro del rango positivo de valores. Es decir, hay un interés medio de la población joven por la situación política del país.

De forma complementaria, al indagar si la juventud tiene un papel importante en el desarrollo del país, se aprecian valores muy elevados, con medias de 3.81 (masculina) y 3.88, (femenina) es decir, sí se evidencia una percepción de la importancia que tiene la juventud para El Salvador.

En contraste, se puede apreciar que en la declaración de que El Salvador da oportunidades a los jóvenes existe una respuesta bastante negativa, siendo sus valores por debajo de la mediana, con 2.28 (masculina) y 2.27 (femenina). En otras palabras, existe una alta percepción de que la juventud es importante, pero al a vez se piensa que a esta misma juventud, que es importante para el desarrollo del país, no se le dan oportunidades de desarrollo en el país.

Siguiendo con la misma cadena de variables, para el ítem de si quiero desarrollarme, tengo que irme del país hay una tendencia positiva a estar de acuerdo, con un 2.92 de hombres y 2.71 de mujeres. Los hombres están más dispuestos a irse que las mujeres, pero ambos grupos se encuentran arriba de la mediana para la escala.

En un tono más vinculado con la ciudadanía nacional, al indagar si existe un conocimiento mínimo de cómo funciona la política del país, las respuestas estuvieron bajo la media, con un 2.31 para los hombres y 2.11 para las mujeres, evidenciando poco entendimiento de cómo se articulan las funciones del Estado y su relación con las instituciones. Por su parte, el entendimiento de los derechos y deberes muestra niveles positivos, con 3.11 y 3.28 para derechos y 3.22 y 3.31 para deberes en ambos casos (hombres y mujeres, respectivamente), siendo las mujeres quienes muestran mayores valores sobre entendimiento $y$ conciencia de sus derechos y deberes ciudadanos.

Finalmente, en cuanto a actitud, se indagó si los participantes se consideran personas que pueden contribuir al desarrollo del país, mostrando, para ambos grupos, una media de 3.88 , es decir, una fuerte idea de que así es el caso.

Tabla 3.

Variables de cultura ciudadana según sexo

\begin{tabular}{|c|c|c|c|c|c|}
\hline \multicolumn{6}{|c|}{ Estadísticos de grupo } \\
\hline & Sexo & $\mathbf{N}$ & Media & Desviación típ. & Error típ. de la media \\
\hline \multirow{2}{*}{ Me interesa la situación política del país } & Masculino & 484 & 3.31 & 1.545 & .070 \\
\hline & Femenino & 472 & 3.03 & 1.480 & .068 \\
\hline \multirow{2}{*}{ La juventud tiene un papel importante en el desarrollo del país } & Masculino & 476 & 3.81 & 1.411 & .065 \\
\hline & Femenino & 480 & 3.88 & 1.387 & .063 \\
\hline \multirow{2}{*}{ El Salvador da oportunidades para los jóvenes } & Masculino & 456 & 2.28 & 1.395 & .065 \\
\hline & Femenino & 484 & 2.27 & 1.373 & .062 \\
\hline \multirow{2}{*}{ Si quiero desarrollarme, tengo que irme del país } & Masculino & 468 & 2.92 & 1.502 & .069 \\
\hline & Femenino & 472 & 2.71 & 1.447 & .067 \\
\hline \multirow{2}{*}{ Sé cómo funciona la política nacional } & Masculino & 464 & 2.31 & 1.297 & .060 \\
\hline & Femenino & 472 & 2.11 & 2.184 & .101 \\
\hline \multirow{2}{*}{ Entiendo los derechos de los ciudadanos } & Masculino & 464 & 3.11 & 1.402 & .065 \\
\hline & Femenino & 464 & 3.28 & 1.346 & .062 \\
\hline \multirow{2}{*}{ Entiendo los deberes de los ciudadanos } & Masculino & 456 & 3.22 & 1.445 & .068 \\
\hline & Femenino & 464 & 3.31 & 1.311 & .061 \\
\hline \multirow{2}{*}{ Soy una persona que puede contribuir al desarrollo del país } & Masculino & 476 & 3.88 & 1.393 & .064 \\
\hline & Femenino & 464 & 3.88 & 1.354 & .063 \\
\hline
\end{tabular}


Al realizar la prueba de hipótesis respectiva se aprecia que existe significancia para el sexo respecto a los ítems de me interesa la situación política del país $(\mathrm{p}=0.04)$, y para si quiero desarrollarme, debo irme del país $(\mathrm{p}=0.024)$, es decir, estadísticamente es posible predecir que a los hombres les interesa un poco más la situación política del país que a las mujeres, de la misma forma en que es más probable que un hombre quiera migrar para tener más oportunidades, mientras que las mujeres, por su parte, muestran valores más bajos a este respecto.

Tabla 4.

Prueba de hipótesis para escala de participación ciudadana según sexo

\begin{tabular}{|c|c|c|c|c|c|c|}
\hline & & \multicolumn{2}{|c|}{$\begin{array}{l}\text { Prueba de Levene para la } \\
\text { igualdad de varianzas }\end{array}$} & \multicolumn{3}{|c|}{ Prueba T para la igualdad de medias } \\
\hline & & $\mathrm{F}$ & Sig. & $t$ & $\mathrm{gl}$ & $\begin{array}{c}\text { Sig. } \\
\text { (bilateral) }\end{array}$ \\
\hline \multirow[t]{2}{*}{ política del país } & $\begin{array}{l}\text { Se han asumido varianzas } \\
\text { iguales }\end{array}$ & 6.335 & .012 & 2.862 & 954 & .004 \\
\hline & $\begin{array}{l}\text { No se han asumido } \\
\text { varianzas iguales }\end{array}$ & & & 2.864 & 953.703 & .004 \\
\hline $\begin{array}{l}\text { Si quiero desarrollarme, } \\
\text { tengo que irme del país }\end{array}$ & $\begin{array}{l}\text { Se han asumido varianzas } \\
\text { iguales }\end{array}$ & 1.877 & .171 & 2.257 & 938 & .024 \\
\hline & $\begin{array}{l}\text { No se han asumido } \\
\text { varianzas iguales }\end{array}$ & & & 2.257 & 936.014 & .024 \\
\hline
\end{tabular}

Al hacer comparación por área de vivienda, se aprecian diferencias importantes entre sectores. En cuanto a formar parte de una subcultura, ambos niveles son bajos; pero los habitantes del área urbana muestran un nivel un poco más elevado que el de los participantes del área rural. 
Tabla 5.

Variables vinculadas con cultura y cosmovisión ciudadana según área de vivienda

\begin{tabular}{|c|c|c|c|c|c|}
\hline & Área de vivienda & $\mathrm{N}$ & Media & Desviación típ. & Error típ. de la media \\
\hline \multirow{2}{*}{ Formo parte de una subcultura } & Urbana & 444 & 1.50 & 1.147 & .054 \\
\hline & Rural & 428 & 1.39 & 1.013 & .049 \\
\hline Mi grupo social participa & Urbana & 460 & 1.78 & 1.251 & .058 \\
\hline $\begin{array}{l}\text { en actividades políticas y } \\
\text { ciudadanas }\end{array}$ & Rural & 436 & 1.74 & 1.193 & .057 \\
\hline Leo el periódico para enterarme & Urbana & 476 & 2.68 & 1.479 & .068 \\
\hline de las noticias & Rural & 456 & 3.04 & 1.543 & .072 \\
\hline Veo los noticieros para & Urbana & 480 & 3.40 & 1.464 & .067 \\
\hline enterarme de las noticias & Rural & 464 & 3.65 & 1.500 & .070 \\
\hline Escucho la radio para enterarme & Urbana & 472 & 2.59 & 1.504 & .069 \\
\hline de las noticias & Rural & 456 & 2.88 & 1.553 & .073 \\
\hline Uso las redes sociales para & Urbana & 484 & 3.21 & 1.627 & .074 \\
\hline esparcimiento & Rural & 452 & 3.12 & 1.557 & .073 \\
\hline Uso las redes sociales con & Urbana & 468 & 3.30 & 1.620 & .075 \\
\hline propósitos informativos & Rural & 464 & 2.16 & 1.634 & .076 \\
\hline
\end{tabular}

De igual manera, otras variables que muestran diferencias bastante pronunciadas entre ambos grupos son el uso de redes sociales, donde el área urbana domina a la rural con más de un punto de diferencia. Es interesante notar que para leer el periódico es el área rural la que manifiesta tener una mayor práctica, en oposición al área urbana, donde probablemente se desarrollan otras formas de información, como las redes sociales.

Al realizar la prueba de hipótesis respectiva, se observa que la única variable que aparece con incidencia significativa respecto al área de vivienda es la de uso de redes sociales, tanto para esparcimiento como para informarse. En cuanto a esparcimiento, se observa que existe una significancia normal $(p=0.004)$, mientras que para el caso de las redes sociales con propósitos informativos existe una significancia todavía más alta, con coeficiente de alfa en p de .000 .

Esto quiere decir que, si una persona vive en el área urbana, será mucho más probable que se informe y divierta a través de las redes sociales que las personas que viven en la rural, probablemente debido a las dificultades de acceso que tienen estas a la red. 
Tabla 6.

Prueba de significancia estadística para variables de cultura ciudadana

Prueba de Levene para la igualdad

de varianzas
Prueba T para la igualdad de medias

\begin{tabular}{|c|c|c|c|c|c|c|}
\hline & & $\mathbf{F}$ & Sig. & $\mathrm{t}$ & gl & Sig. (bilateral) \\
\hline Uso las redes sociales & Se han asumido & & & & & \\
\hline \multirow[t]{2}{*}{ para esparcimiento } & varianzas iguales & 11.256 & .001 & 2.772 & 778 & .004 \\
\hline & $\begin{array}{l}\text { No se han asumido } \\
\text { varianzas iguales }\end{array}$ & & & 2.921 & 332.211 & .004 \\
\hline \multirow{5}{*}{$\begin{array}{l}\text { Uso las redes sociales } \\
\text { con propósitos } \\
\text { informativos }\end{array}$} & Se han asumido & & & & & \\
\hline & varianzas iguales & 19.560 & .000 & 1.585 & 774 & .000 \\
\hline & & & & & & \\
\hline & $\begin{array}{l}\text { No se han asumido } \\
\text { varianzas iquales }\end{array}$ & & & & & \\
\hline & varianzas iguales & & & 1.688 & 339.845 & .002 \\
\hline
\end{tabular}

Para las variables de interés en la política y cultura democrática respecto al tipo de educación básica recibida, se aprecian grandes diferencias entre grupos. Aquellos que manifiestan haber salido de un centro escolar privado tienen medias mayores en casi todas las áreas, en comparación con aquellos que provienen de centros públicos. En contraste, ante las variables vinculadas con competencias de participación, se observa que aquellos provenientes del sector privado poseen una mayor cantidad de competencias en comparación con los del sector público. Para la variable de sé cómo funciona la política nacional, el sector público obtuvo una media de 2.35, mientras que el privado sacó una de 2.71, es decir, por parte de este sector existe una mejor comprensión del funcionamiento del Estado, probablemente vinculada con el tipo de educación recibida en Estudios Sociales. 
Tabla 7.

Medias de contraste según sector de centro de estudios

\begin{tabular}{|c|c|c|c|c|c|}
\hline & $\begin{array}{l}\text { Sector de } \\
\text { estudios }\end{array}$ & $\mathbf{N}$ & Media & $\begin{array}{c}\text { Desviación } \\
\text { típ. }\end{array}$ & $\begin{array}{l}\text { Error típ. de } \\
\text { la media }\end{array}$ \\
\hline \multirow{2}{*}{ Me interesa la situación política del país } & Público & 608 & 3.13 & 1.554 & .063 \\
\hline & Privado & 184 & 3.80 & 1.299 & .096 \\
\hline \multirow{2}{*}{ La juventud tiene un papel importante en el desarrollo del país } & Público & 608 & 3.84 & 1.408 & .057 \\
\hline & Privado & 184 & 4.04 & 1.182 & .087 \\
\hline \multirow{2}{*}{ El Salvador no da oportunidades para los jóvenes } & Público & 604 & 2.87 & 1.455 & .059 \\
\hline & Privado & 180 & 2.49 & 1.006 & .075 \\
\hline \multirow{2}{*}{ Si quiero desarrollarme, tengo que irme del país } & Público & 608 & 2.64 & 1.499 & .061 \\
\hline & Privado & 180 & 2.20 & 1.296 & .097 \\
\hline \multirow{2}{*}{ Sé cómo funciona la política nacional } & Público & 600 & 2.35 & 2.058 & .084 \\
\hline & Privado & 180 & 2.71 & 1.143 & .085 \\
\hline \multirow{2}{*}{ Entiendo los derechos de los ciudadanos } & Público & 588 & 3.23 & 1.382 & .057 \\
\hline & Privado & 180 & 3.60 & 1.044 & .078 \\
\hline \multirow{2}{*}{ Entiendo los deberes de los ciudadanos } & Público & 592 & 3.30 & 1.394 & .057 \\
\hline & Privado & 180 & 3.62 & 1.104 & .082 \\
\hline \multirow{2}{*}{ Soy una persona que puede contribuir al desarrollo del país } & Público & 596 & 3.95 & 1.351 & .055 \\
\hline & Privado & 184 & 4.33 & .959 & .071 \\
\hline
\end{tabular}

\section{Discusión}

El trabajo de investigación mostró hallazgos importantes en cuanto a las variables que inciden en el desarrollo del imaginario colectivo vinculado con la participación ciudadana y democrática. De acuerdo con Padilla (2002), todo Estado democrático moderno opera en multiplicidad de dominios políticos e interactúa con la ciudadanía a través de una amplia diversidad de agencias e instituciones. Esto significa que el ideal de "igualdad de derechos" tiene que ser interpretado, filtrado y transmitido a través de un enorme conjunto de canales que, en su mayor parte, están fundamentalmente centrados en tareas especializadas y tienen contacto directo con solo una pequeña parte del electorado total. En base a este principio, podemos apreciar que los elementos de cultura ciudadana están vinculados con la educación como principio básico, condicionando la igualdad al conocimiento de los elementos que determinan el imaginario colectivo de la juventud respecto a los temas de política y ciudadanía.
En el ámbito general se aprecia que existe una concepción de que los jóvenes son importantes para el desarrollo democrático del país, pero esta a su vez contrasta con el hecho de que hay una fuerte percepción de que el país no da oportunidades a los jóvenes. Esto, aunado a la idea de que, para desarrollarse, un joven debe irse del país, manifiesta que la exclusión social, particularmente la vinculada con los jóvenes, todavía está presente y con consecuencias negativas para el desarrollo democrático del país.

En contraste, la educación nuevamente muestra ser un fenómeno transversal a la ciudadanía, puesto que existe autopercepción de un conocimiento medio-alto acerca de los derechos y deberes que los jóvenes tienen como ciudadanos, pero al ser más específicos e indagar elementos de la cultura ciudadana, prácticas como leer el periódico y utilizar la tecnología para informarse salen relativamente bajas, utilizándose las redes sociales para entretenimiento más que para conseguir información, siendo las jóvenes las que utilizan este medio para esto último. 
Al hacer la misma comparación por área de vivienda, se observa que las principales variables afectadas son uso de redes sociales, el cual aparece significativamente más bajo en el área rural para información, pero en el mismo nivel que el área urbana para entretenimiento, lo que evidencia que, más que una dificultad de acceso a este recurso, se trata de la educación sobre el uso de este para un propósito que permita acceder a una participación social activa. Para la variable de interés en la política y cultura democrática respecto al tipo de educación básica recibida, se aprecian grandes diferencias entre grupos. Aquellos que manifiestan haber salido de un centro escolar privado tienen medias mayores en casi todas las áreas, en comparación con aquellos que provienen de un centro público. Es decir, el tipo de educación recibida tiene una incidencia bastante fuerte en el conocimiento y la actitud que se tenga hacia la participación política, así como el tipo de cultura ciudadana e imaginario político que se desarrolle. En pocas palabras, la educación es el pilar fundamental de una participación ciudadana coherente, así como de la cultura política y la adquisición de conocimientos vinculados con la cultura ciudadana, que permita conocimiento de las instituciones, uso correcto de estas, reducción de las prácticas discriminativas, pertenencia a grupos sociales de mayor incidencia en la cotidianidad social, mejores prácticas informativas y participativas, entre otros aspectos. Esto, aunado al ya existente sentimiento de que la juventud es importante para el desarrollo democrático del país, permitiría una verdadera inserción de los jóvenes a la democracia del país no en espacios fabricados para incidir en elementos políticos específicos dados por las instituciones, sino en cualquier ámbito de la vida social para proponer y cambiar.

\section{Referencias}

Campos Morán, S. (2011). “Formación ciudadana en jóvenes y su impacto en el proceso democrático del país". Universidad Tecnológica de El Salvador, Utec, San Salvador.

Encuesta Nacional de Hogares de Propósitos Múltiples (2013). Dirección Nacional de Estadística y Censos. Disponible en http://www.digestyc.gob.sv/index. php/temas/des/ehpm.html

Fernández, M. (2001). "La sociedad en el marco de las políticas educativas". Sociedad Educadora, Revista Iberoamericana de Educación. http://www.rieoei. org/rie26a08.htm

Montero, O.; León, I. (2007). "A guide for naming research studies in psychology". International Journal of Clinical and Health Psychology. ISSN 1697-2600

Padilla, G. (2002). "Juventud y cultura política". Flacso, Programa El Salvador. ISBN 99923-33-20-0.

Peláez, J. (2008) "Salud sexual y reproductiva de adolescentes y jóvenes: una mirada desde la óptica de los derechos humanos". Disponible en http://bvs. sld.cu/revistas/gin/vol34_2_08/gin05208.htm

Tribunal Supremo Electoral (2014). Elecciones Presidenciales 2014. Disponible en http://www.tse.gob.sv/ elecciones/elecciones-2014

Vuanello, R. (2007). "Inseguridad urbana y trauma: jóvenes en riesgo". http://psicologiajuridica.org/psj217.html 